\title{
Differences according to Sex in Sociosexuality and Infidelity after Traumatic Brain Injury
}

\author{
Jhon Alexander Moreno ${ }^{1,2}$ and Michelle McKerral ${ }^{1,2}$ \\ ${ }^{1}$ Center for Interdisciplinary Research in Rehabilitation (CRIR), Centre de Réadaptation Lucie-Bruneau (CRLB), \\ 2275 Laurier Avenue East, Montréal, QC, Canada H2H 2N8 \\ ${ }^{2}$ Centre de Recherche en Neuropsychologie et Cognition (CERNEC), Département de Psychologie, Université de Montréal, \\ Montréal, QC, Canada \\ Correspondence should be addressed to Jhon Alexander Moreno; jhon.alexander.moreno@umontreal.ca and \\ Michelle McKerral; michelle.mckerral@umontreal.ca
}

Received 21 July 2015; Accepted 23 August 2015

Academic Editor: Hrayr Attarian

Copyright (C) 2015 J. A. Moreno and M. McKerral. This is an open access article distributed under the Creative Commons Attribution License, which permits unrestricted use, distribution, and reproduction in any medium, provided the original work is properly cited.

Objective. To explore differences according to sex in sociosexuality and infidelity in individuals with TBI and in healthy controls. Participants. Forty-two individuals with mild, moderate, and severe TBI having completed a postacute TBI rehabilitation program, at least six months after injury, and 47 healthy controls. Main Measures. Sociosexual Orientation Inventory-Revised (SOI-R) and Attitudes toward Infidelity Scale. Results. Overall, men score significantly higher than women in sociosexuality. However, there was a nonsignificant trend towards a reduction of sociosexuality levels in men with TBI. Infidelity levels were comparable in healthy controls and individuals with TBI. In individuals with TBI, less acceptance of infidelity was significantly associated with an unrestricted sociosexual orientation, but not in healthy controls. Conclusions. As documented in previous cross-cultural studies, men have higher levels of sociosexuality than women. However, men with TBI showed a tendency towards the reduction of sociosexuality. The possibility of a latent explanatory variable is suggested (e.g., post-TBI neuroendocrinological changes). TBI does not seem to have an impact on infidelity, but individuals with TBI who express less acceptance of infidelity also report a more promiscuous mating strategy regarding their behavior, attitudes, and desire. Theoretical implications are discussed in terms of evolutionary theories of human sexuality and neuropsychology.

\section{Introduction}

Nonmonogamy is part of the evolutionary trends preserved in humans [1]. In fact, infidelity constitutes probably one of the most complex problems faced by mental health professionals, especially couple therapists, marriage and family therapists, and psychotherapists $[2,3]$. Based on evolutionary theories, there are sex differences regarding reactions to infidelity. For instance, men seem to be more distressed by sexual infidelity (e.g., sexual relationship or sexually oriented physical contact with another person), while women may be more distressed by emotional infidelity (e.g., diversion of the partner's emotional commitment toward another person) [4]. Interestingly, human brains show different activation patterns in response to different types of infidelity; men and women process sexual and emotional infidelity using different neuropsychological networks [5].

Nonetheless, the experience of infidelity is linked to the individuals' proneness to be unfaithful. This is the research area of sociosexuality, also known as sociosexual orientation (SO). Kinsey was the first to introduce the term in his pioneer studies describing individual differences in people's willingness to engage in uncommitted sexual relationships $[6,7]$. Sociosexuality levels range from an unrestricted SO to a restricted SO. Individuals with an unrestricted SO tend to a more promiscuous mating strategy, are quicker to have sex, and may experience lower levels of romantic relationship closeness or commitment. Conversely, individuals with 
a restricted SO tend to a more monogamous mating approach, invest more time in courtship, and develop strong emotional connections in long-term relationships [8].

Undoubtedly, the most striking cross-cultural evidence of the existence of SO comes from the international sexuality description project [9]. This groundbreaking analysis of sociosexuality in 48 nations demonstrated that, compared to women, men have higher levels of sociosexuality across cultures and that sex differences in sociosexuality are culturally universal. Furthermore, even though sex differences in sociosexuality are attenuated in cultures with more gender equality in terms of political, economic, and relational freedom, the findings of this study did not suggest that men and women tend to become equally promiscuous in attitudes and behaviors. Research has demonstrated the existence of a sociosexuality-testosterone association in both men and women and revealed that the nature of these associations varies by gender and relationship status (e.g., partnered men who reported an unrestricted sociosexuality had testosterone levels that were comparable to those of single men) [10]. However, research in the area of sociosexuality has included not only differences according to sex [11] but also many other variables, such as racial differences [12], infidelity [13], attachment style [4], self-image [14], physical attractiveness and sexual aggression perpetration [15], and personality styles [16], among others.

Overall, the aforementioned studies highlight the importance of sociosexuality, from both an evolutionary and an environmental/sociocultural perspective to explain the reasons behind the fact that, on average, men are more willing than women to engage in casual sex. Two different interpretations have been suggested. In his seminal theory of parental investment and sexual selection, Trivers (1973) defined parental investment as the resources that a parent spends on his offspring in order to increase the chances of surviving and reproducing, at the cost of this parent's ability to invest in other offspring [17-19]. Together, these studies outline that, from an evolutionary perspective, men have more to gain and less to lose by having sex outside a committed relationship. In contrast, women have to invest time and energy devoted to pregnancy and childbearing. This interpretation contrasts with an environmental/sociocultural perspective, suggesting the possibility that differences in sociosexuality can be associated, in part, with the variations in the regional prevalence of infectious diseases. People in regions with a history of a high prevalence of infectious diseases report lower levels of sociosexuality [20].

Given the evolutionary, neuropsychological, and environmental/sociocultural rationales put forward in the research literature on sociosexuality, its presentation in acquired medical conditions where changes in brain functions are induced warrants investigation. Traumatic brain injury (TBI), which is among the most common neurological conditions [21], is a form of brain injury which is receiving increasing attention in the area of research on sexuality, given its biopsychosocial consequences [22-27].

Also, TBI impacts people's sexuality, with 50 to $60 \%$ of persons reporting some level of disruption after injury [2830], and sexual function is compromised as a result of the
post-TBI changes involving the neurological aspects of sexuality [27]. Sexual difficulties after TBI have thus been associated with medical and physical issues (e.g., neuroendocrine and hormonal disorders [31, 32], neuropsychological and psychological effects (e.g., depression [33]), and relationship changes (e.g., intimacy [34])) [25].

To our knowledge, previous studies on sexuality and TBI have not addressed attitudes towards infidelity and sociosexuality in individuals with TBI. The current study thus aimed to explore differences according to sex in sociosexuality and attitudes towards infidelity in individuals with TBI and in healthy controls. In the current study, infidelity is defined as a person being unfaithful while in a committed monogamous relationship. Since this is a novel and exploratory study, no specific hypotheses related to TBI participants are advanced, but it is postulated that there are statistically significant differences according to sex in sociosexuality for healthy controls, with men having higher levels of SO compared to women.

\section{Methods}

2.1. Participants. The sample consisted of 42 individuals with TBI and 47 healthy controls. Individuals with TBI were recruited from a TBI outpatient rehabilitation center in Montreal, which offers social and vocational rehabilitation services to individuals with moderate or severe TBI, as well as to individuals with mild or complex mild TBI showing atypical recovery to which the brain injury appears to contribute predominantly. Individuals with TBI were recruited based on the following inclusion criteria: (1) individuals who have sustained, according to the TBI guidelines put forward by the Québec Ministry of Health [35], a mild (Glasgow coma scale (GCS) scores 13-15), moderate (GCS scores 9-12), or severe (GCS scores 3-8) TBI, (2) individuals who are six or more months post-injury, (3) individuals who are 18 years or older, and (4) individuals who report to be able to read, write, and speak either French or English. Exclusion criteria, as verified in medical records, included (1) history of learning or language disability, including aphasia or communication disorders and (2) self-report of preinjury psychiatric, sexual, or neurological disorders other than TBI. A detailed description of the sociodemographic characteristics of the sample is provided in Table 1.

In terms of clinical characteristics, as indicated in Table 2, the majority corresponds to mild TBIs (66.8\%). The cause of the injury was predominantly associated with a motor vehicle accident (42.9\%) followed by work and sports-related accidents (14.3\%). Half of them had a history of loss of consciousness (50\%) and $47.6 \%$ had also a history of posttraumatic amnesia documented in the medical chart. Individuals with TBI were on average 3.3 years after the injury $(\mathrm{SD}=4.3)$. Positive findings on CT scan or MRI suggesting a brain injury were documented in 59.5\%. Glasgow coma scale at admission was on average $12.5(\mathrm{SD}=3.6)$, with a loss of consciousness of a mean of 5.8 hours $(\mathrm{SD}=28.8)$ and posttraumatic amnesia duration of 80.8 hours $(S D=203.8)$ as indicated in medical records. 
TABLE 1: Sociodemographic characteristics of the TBI and healthy control samples $(N=89)$.

\begin{tabular}{|c|c|c|c|c|}
\hline & TBI & Healthy controls & TBI & Healthy controls \\
\hline & \multicolumn{2}{|c|}{ Frequency $(\%)$} & \multicolumn{2}{|c|}{ Mean $(\mathrm{SD})$} \\
\hline \multicolumn{5}{|l|}{ Gender } \\
\hline Male & $19(45.2 \%)$ & $24(51.1 \%)$ & & \\
\hline Female & $23(54.8 \%)$ & $23(48.9 \%)$ & & \\
\hline \multicolumn{5}{|l|}{ Race and ethnicity } \\
\hline White & $38(90.5 \%)$ & $45(95.7 \%)$ & & \\
\hline Hispanic & $4(9.5 \%)$ & $2(4.3 \%)$ & & \\
\hline \multicolumn{5}{|l|}{ Work status } \\
\hline Full time & $16(38.1 \%)$ & $26(55.3 \%)$ & & \\
\hline Part time & $7(16.7 \%)$ & $7(14.9 \%)$ & & \\
\hline Unemployed & $19(45.2 \%)$ & $13(27.7 \%)$ & & \\
\hline Missing & $0(0 \%)$ & $1(2.1 \%)$ & & \\
\hline \multicolumn{5}{|l|}{ Relationship status } \\
\hline Single & $26(61.9 \%)$ & $21(44.7 \%)$ & & \\
\hline Married & $4(9.5 \%)$ & $5(10.6 \%)$ & & \\
\hline Separated & $0(0 \%)$ & $4(8.5 \%)$ & & \\
\hline Divorced & $2(4.8 \%)$ & $2(4.3 \%)$ & & \\
\hline Common-law & $10(23.8 \%)$ & $14(29.8 \%)$ & & \\
\hline Widow/widower & $0(0 \%)$ & $1(2.1 \%)$ & & \\
\hline Age (years) & & & $37.9(9.7)$ & $37.6(10.7)$ \\
\hline Education (years) & & & $12.8(3.3)$ & $13(3.0)$ \\
\hline Annual income (CAD) & & & $39007.5(19239.6)$ & $31975.6(18909.9)$ \\
\hline
\end{tabular}

Note. CAD, Canadian dollars.

Healthy controls were recruited from the community following these inclusion criteria: (1) being 18 years or older and (2) reporting to be able to read, write, and speak either French or English. Exclusion criteria included (1) selfreported history of learning or language disability and (2) self-report of diagnosed psychiatric, sexual, or neurological disorders. Their sociodemographic characteristics are presented in Table 1.

2.2. Procedure. The current study was approved by the Research Ethics Board (REB) of the Center for Interdisciplinary Research in Rehabilitation of Greater Montreal (CRIR). Data collection was undertaken between April 2013 and August 2014.

From the rehabilitation center's database, a total of 345 individuals with TBI were eligible for participation. Following telephone contact by a person independent of the research project (e.g., archives technician) who proposed participation in the study, 13 of them refused to participate and 224 could not be reached. Individuals with TBI who accepted to participate were mailed two envelopes: (a) a consent form (which included a thorough explanation of the study) and (b) a package containing the questionnaires. Each of the envelopes contained a stamped and addressed envelope so that the participant could return each document independently. Questionnaires and consent forms were sent to 108 individuals with TBI and 42 of them successfully completed and returned both (41 in French and 1 in English).
In the context of a larger sexuality study, healthy controls were recruited from the general community through newspaper advertisements, as well as notices in community centers, universities, and libraries. A total of 242 people from the community expressed their interest to participate in the sexuality study. Following a phone call by the research team to verify inclusion/exclusion criteria, questionnaires and consent forms were sent to 191 healthy controls. Twentyeight of them did not return both the questionnaires and consent forms while 163 returned them. For the purposes of this study, 47 healthy controls ( 41 in French and 6 in English) were matched to TBI participants from the database of the aforementioned large sexuality study, based on sociodemographic variables (e.g., age, gender, years of education, annual income, work, and relationship status). Questionnaire data were subsequently analyzed.

Voicemail and email accounts were created in order to receive and answer any questions for individuals with TBI or healthy controls. All participants received a financial compensation of CAN $\$ 15$ (fifteen Canadian dollars) for their participation after returning their questionnaires and consent forms.

\subsection{Instruments}

2.3.1. Medical History and Demographic Information. Participants completed an in-house short medical and sociodemographic questionnaire that included questions related 
TABLE 2: Clinical characteristics of the sample of individuals with TBI $(N=42)$.

\begin{tabular}{|c|c|c|}
\hline & Frequency $(\%)$ & Mean (SD) \\
\hline \multicolumn{3}{|l|}{ Cause of the injury } \\
\hline Motor vehicle accident & $18(42.9 \%)$ & \\
\hline Violence & $2(4.8 \%)$ & \\
\hline Falls & $4(9.5 \%)$ & \\
\hline Sports-related & $6(14.3 \%)$ & \\
\hline Work accident & $6(14.3 \%)$ & \\
\hline Other & $3(7.1 \%)$ & \\
\hline Missing & $3(7.1 \%)$ & \\
\hline \multicolumn{3}{|l|}{ LOC } \\
\hline Yes & $21(50 \%)$ & \\
\hline No & $18(42.9 \%)$ & \\
\hline Missing & $3(7.1 \%)$ & \\
\hline \multicolumn{3}{|l|}{ PTA } \\
\hline Yes & $20(47.6 \%)$ & \\
\hline No & $19(45.3 \%)$ & \\
\hline Missing & $3(7.1 \%)$ & \\
\hline \multicolumn{3}{|l|}{ Positive CAT or MRI } \\
\hline Yes & $25(59.5 \%)$ & \\
\hline No & $10(23.8 \%)$ & \\
\hline Missing & $7(16.7 \%)$ & \\
\hline \multicolumn{3}{|l|}{ Severity of the injury } \\
\hline Mild TBI & $28(66.8 \%)$ & \\
\hline Moderate TBI & $3(7.1 \%)$ & \\
\hline Severe TBI & $8(19 \%)$ & \\
\hline Missing & $3(7.1 \%)$ & \\
\hline GCS & & $12.5(3.6)$ \\
\hline Years after injury & & $3.3(4.3)$ \\
\hline Length of LOC (hours) & & $5.8(28.8)$ \\
\hline Length of PTA (hours) & & $80.8(203.8)$ \\
\hline
\end{tabular}

Note. LOC, loss of consciousness; PTA, posttraumatic amnesia; CAT, computed axial tomography; MRI, magnetic resonance imaging; and GCS, Glasgow coma scale.

to participant's age (e.g., number of years), race/ethnicity (e.g., white, Hispanic), gender (e.g., male, female), years of education (e.g., number of years), relationship status (e.g., single, married), annual income (in Canadian dollars), work status (e.g., full time, unemployed), frequency of alcohol (e.g., never to everyday), and recreational drug use (e.g., yes, no). For TBI participants, data regarding preinjury and injury related variables (e.g., severity of injury, number of years after injury, length of loss of consciousness in hours, length of posttraumatic amnesia in hours, and presence/absence of neuroradiological abnormalities) were extracted from medical records.

Each of the participants was administered the following questionnaires.

Sociosexual Orientation Inventory-Revised (SOI-R). The SOI$\mathrm{R}$ is a 9-item self-report questionnaire, each with a 9-point response scale, developed to measure individual differences in willingness to engage in casual, uncommitted sexual relationships [8]. In particular, the SOI-R assesses individual's past behavior in terms of number of casual and changing sex partners, the explicit attitude towards uncommitted sex, and sexual desire for people with whom no romantic relationship exists [36]. Scores for behavior, attitude, and desire facets as well as a total score are obtained [37]. Higher scores on the SOI-R correspond to individuals who have an unrestricted sociosexual orientation (or have a more promiscuous mating strategy) whereas lower scores correspond to restricted sociosexual orientation (or individuals who follow a more monogamous mating strategy). The SOI-R proposes adequate reliability and validity both within and across the diverse range of human cultures [9] and has been used widely in a variety of research and clinical samples [37-44]. For items 1 to 3 , values of 1 to 9 should be assigned to the responses. Thus, all nine items have values from 1 to 9 (9-point scale). Item 6 should be reverse-keyed. Items 1 to 3 are aggregated (summed or averaged) to form the behavior facet, items 4 to 6 form the attitude facet, and items 7 to 9 form the desire facet. Finally, all nine items can be aggregated to form a full-scale score that represents the global SO. In the current study, the internal consistency of SOI-R (Cronbach's $\alpha=0.89$ ), as well as all of the three facets of the SOI-R, was very good (behavior Cronbach's $\alpha=0.91$, attitude Cronbach's $\alpha=0.84$, and desire Cronbach's $\alpha=0.88$ ).

Attitudes toward Infidelity Scale. This is a 12-item self-report questionnaire to measure the acceptance of infidelity. In the context of this scale, infidelity is defined as a person being unfaithful in a committed monogamous relationship. Each item is rated on a 7-point Likert scale with 1 reflecting the least acceptance of infidelity and 7 the greatest acceptance of infidelity. The lower the total score (12 is the lowest possible score), the less the person's acceptance of infidelity, whereas the higher the total score ( 84 is the highest possible score), the greater the respondent's acceptance of infidelity [45]. A score of 48 places the person at the midpoint between being very disapproving of infidelity and very accepting of infidelity. Before adding the numbers, score items $2,5,6,7,8$, and 12 must be reversed (e.g., $1=7,2=6,3=5,4=4,5=3,6=2$, and $7=1$ ). After making these changes, the numbers must be added to obtain the full-scale score [46]. A translation/backtranslation procedure was implemented in order to obtain the French version that was used in the present study and its internal consistency was good (Cronbach's $\alpha=0.79$ ).

2.4. Statistical Analyses. Demographic characteristics of individuals with TBI were compared to those of healthy controls using $t$-tests for continuous variables and $\chi^{2}$ tests for nominal variables, taking into account a significance level $p<0.05$.

Two-way between-groups analyses of variance (two-way ANOVA) were performed to explore the impact of sex (e.g., male and female) and group (e.g., individuals with TBI and healthy controls) on sociosexuality.

An independent-samples $t$-test was performed to compare infidelity levels between individuals with TBI and healthy controls. Pearson correlation analyses were used 
to examine the relationship between sociosexuality facets (behavior, attitude, and desire), infidelity levels, and injury characteristics (years after injury, GCS score, and hours of posttraumatic amnesia) in individuals with TBI.

Statistical analyses were conducted with IBM SPSS version 21 [47].

\section{Results}

Comparison of the sociodemographic characteristics of the TBI and healthy control groups, described in Table 1, indicates that there were no significant differences between groups in terms of age, gender, race/ethnicity, work status, relationship status, years of education, and annual income. Also, both groups were comparable in frequency of alcohol consumption, recreational drug use, and the use of one prescribed medication. Comparison of the sociodemographic and clinical characteristics of the TBI group by gender indicates that there were no significant differences between men and women with TBI in terms of age, race/ethnicity, work status, relationship status, years of education, annual income, alcohol consumption, recreational drug use, medication intake, injury severity, time after injury, neuroimaging evidence of brain injury, or loss of consciousness/posttraumatic amnesia duration (all $p$ 's $>0.05$ ).

As summarized in Table 3, a two-way between-groups analysis of variance was performed to explore the impact of sex (male-female) and group (individuals with TBI and healthy controls) on sociosexuality, as measured by the SOI$\mathrm{R}$. The interaction effect between sex and group was not statistically significant, $F(1,85)=0.6, p>0.05$. There was a statistically significant main effect for sex, $F(1,85)=7.2$, $p<0.05$; and the effect size was in the range of medium to large effect size (partial eta squared $=0.07$ ) according to the guidelines for the behavioral sciences [48]. The main effect for group, $F(1,85)=1.0, p>0.05$, did not reach statistical significance. Compared to females, overall, males had higher levels of sociosexuality. However, there appeared to be a tendency towards a reduction of sociosexuality levels in males with TBI (see Figure 1).

Finally, compared to healthy controls, individuals with TBI did not show statistically significant differences in infidelity, as measured by the total score of the Attitudes toward Infidelity Scale, $t(85)=-0.8, p>0.05$.

3.1. Correlation Matrix. The relationship between infidelity (as measured by the Attitudes toward Infidelity Scale), sociosexuality (as measured by the SOI-R), and TBI characteristics (severity as measured by the score on the GCS scale and by length of posttraumatic amnesia in hours, years after injury) in the group of individuals with TBI was investigated using Pearson product-moment correlation coefficient (see Table 4). There was a large negative correlation between the scores on the infidelity scale and the SOI-R $(r=-0.58, p<$ 0.01 ), with low levels of infidelity scores (less permissiveness regarding infidelity) associated with high levels of SO (unrestricted SO). In addition, infidelity scores were moderately associated with behavioral sociosexuality $(r=-0.34, p<$ $0.05)$ and sociosexual desire $(r=-0.49, p<0.01)$. Also,

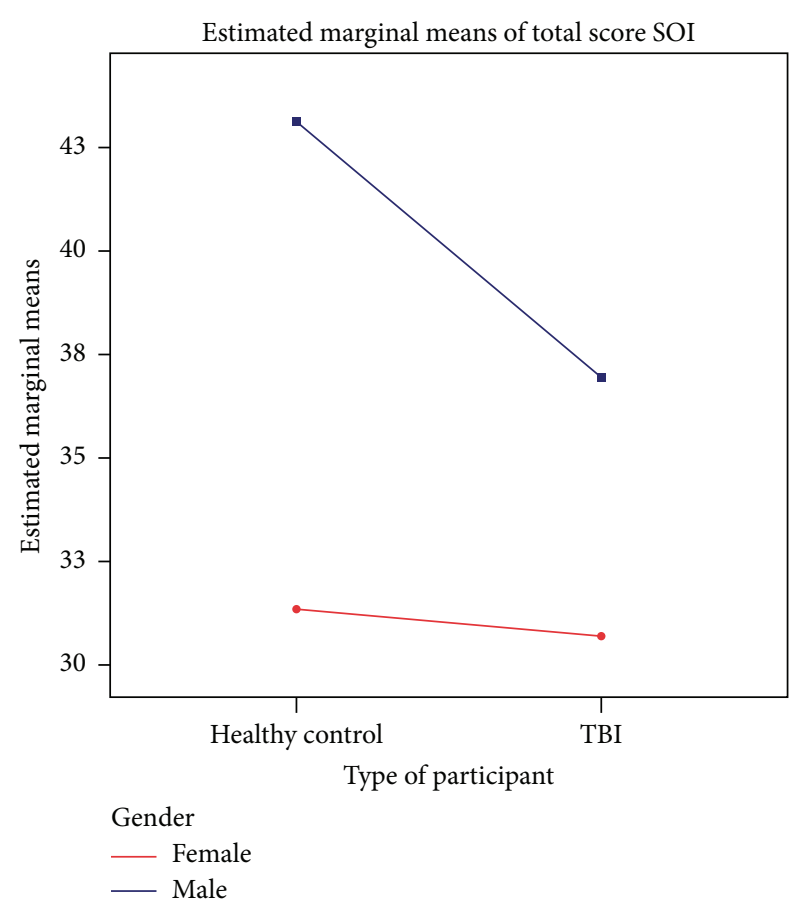

FIGURE 1: Estimated marginal means for sociosexuality as a function of group and gender. Abbreviation: SOI, total score of the Sociosexual Orientation Inventory.

infidelity scores showed a large correlation with sociosexual attitudes $(r=-0.57, p<0.01)$. In contrast, these associations were not significant in the group of healthy controls (all $p$ 's $>$ 0.05).

Finally, neither infidelity scores nor sociosexuality was associated with severity of the injury (GCS score or length of posttraumatic amnesia), or with time since injury (all $p$ 's > 0.05).

\section{Discussion}

The current study aimed to explore differences according to sex in sociosexuality and attitudes towards infidelity in individuals with TBI and healthy controls. The main finding of the current study is that, compared to healthy controls, our TBI sample appeared to show a tendency towards a reduction of differences according to sex in sociosexuality. Interestingly, there was a trend suggesting a decrease in sociosexuality levels in men with TBI. To our knowledge, this study is the first suggesting the possibility of a decline of this crosscultural and evolutionary distinction following TBI in males. This finding is important since it could suggest that a complex and deeply rooted psychosexual trait, such as sociosexuality, could be modified after a neurological insult such as TBI.

The tendency towards the reduction of differences according to sex in sociosexuality levels following TBI does not seem to be explained by sociodemographic or clinical variables. Then, it is possible that a latent variable could account for this trend. From the standpoint of neuropsychology, a possible explanation for this might be 
TABLE 3: Means, standard deviations, and analysis of variance (ANOVA) results for sociosexuality and infidelity as a function of group and sex.

\begin{tabular}{|c|c|c|c|c|c|c|c|}
\hline \multirow{2}{*}{ Measure } & \multicolumn{2}{|c|}{ TBI } & \multicolumn{2}{|c|}{ Healthy controls } & \multicolumn{3}{|c|}{ ANOVA $F$} \\
\hline & M & SD & M & $\mathrm{SD}$ & Group (G) & $\operatorname{Sex}(S)$ & $\mathrm{G} \times \mathrm{S}$ \\
\hline SOI-R & & & & & 1.0 & $7.2^{*}$ & 0.6 \\
\hline Female & 30.7 & 14.8 & 31.3 & 12.9 & & & \\
\hline Male & 36.9 & 19.2 & 43.1 & 16.0 & & & \\
\hline SOI-BEH & & & & & 0.3 & 2.5 & 3.5 \\
\hline Female & 8.2 & 5.0 & 6.6 & 3.0 & & & \\
\hline Male & 7.8 & 7.3 & 11.0 & 7.6 & & & \\
\hline SOI-ATT & & & & & 2.0 & 3.3 & 0.1 \\
\hline Female & 14.1 & 7.8 & 15.7 & 7.2 & & & \\
\hline Male & 16.4 & 7.9 & 19.2 & 6.2 & & & \\
\hline SOI-DES & & & & & 0.1 & $10.3^{*}$ & 0.0 \\
\hline Female & 8.3 & 4.9 & 9.0 & 5.5 & & & \\
\hline Male & 12.7 & 7.9 & 12.9 & 5.9 & & & \\
\hline ATIS & & & & & 0.7 & 0.6 & 0.0 \\
\hline Female & 62.7 & 12.9 & 60.5 & 15.4 & & & \\
\hline Male & 60.7 & 14.8 & 58.0 & 10.9 & & & \\
\hline
\end{tabular}

Note. ${ }^{*} p<0.05$.

SOI-R, total score of the Sociosexual Orientation Inventory-Revised; SOI-BEH, sociosexual behavior; SOI-ATT, sociosexual attitudes; SOI-DES, sociosexual desire; and ATIS, Attitudes toward Infidelity Scale.

TABLE 4: Correlation matrix between infidelity, sociosexuality, and brain injury characteristics.

\begin{tabular}{|c|c|c|c|c|c|c|c|c|}
\hline & & 1 & 2 & 3 & 4 & 5 & 6 & 7 \\
\hline 1 & ATIS & - & & & & & & \\
\hline 2 & SOI-R & $-0.58^{* *}$ & - & & & & & \\
\hline 3 & SOI-BEH & $-0.34^{*}$ & $0.77^{* *}$ & - & & & & \\
\hline 4 & SOI-ATT & $-0.57^{* *}$ & $0.84^{* *}$ & $0.45^{* *}$ & - & & & \\
\hline 5 & SOI-DES & $-0.49^{* *}$ & $0.84^{* *}$ & $0.52^{* *}$ & $0.57^{* *}$ & - & & \\
\hline 6 & GCS & 0.12 & -0.30 & -0.22 & -0.30 & -0.21 & - & \\
\hline 7 & Years after TBI & 0.17 & -0.18 & -0.15 & -0.27 & 0.01 & 0.05 & - \\
\hline 8 & PTA (hours) & -0.01 & 0.18 & 0.31 & 0.12 & 0.02 & $-0.58^{* *}$ & -0.05 \\
\hline
\end{tabular}

Note. ${ }^{*} p<0.05{ }^{* *} p<0.01$.

ATIS, scores of the attitudes toward infidelity scale; SOI-R, total score of the Sociosexual Orientation Inventory-Revised; SOI-BEH, sociosexual behavior; SOIATT, sociosexual attitudes; SOI-DES, sociosexual desire; GCS, Glasgow coma scale; and PTA, posttraumatic amnesia.

the existence of post-TBI neuroendocrine changes. Previous research indicating the existence of a link between testosterone and sociosexuality could represent a basis for such modifications [10]; the effects of neuroendocrine postTBI dysfunction on testosterone levels and its precursors could modify sociosexuality levels. In fact, posttraumatic hypopituitarism is an underdiagnosed complication of TBI [49] and reports indicating that TBI is a common cause of pituitary dysfunction are compelling [50-98]. The main gonadal male hormone is testosterone, which is essential for the development of secondary sexual characteristics and behavioral patterns [99]. In addition, evidence from animal models of sexuality following TBI indicates that TBI-induced hypopituitarism in male rats causes decreased testosterone production and changes in sexual behavior [100]. However, this interpretation must be considered with caution since we did not measure testosterone levels in our study participants. Hence, further research in individuals with TBIs of different severities needs to be conducted to determine if this is an actual contributing cause.

As expected and consistent with previous reports, our results showed that there are statistically significant differences according to sex in sociosexuality. The results of the current study support our hypothesis and add new evidence to the fact that, compared to women, men have higher levels of sociosexuality across cultures [9]. These findings corroborate a great deal of the previous work in the field of sociosexuality $[8,11,14,15,37,101-104]$. The results are also in the same direction of Canadian reports of sexual attitudes and behaviors. Specifically, the results of a Canadian study revealed that, compared to women, men had more frequent sexual thoughts, were more likely to report having engaged in oral sex, had a lower age at first intercourse, had more sexual partners, and were more willing to have casual sex [105].

Theories from evolutionary and comparative psychology bring elements to try to understand the fact that, on average, 
men are more willing than women to engage in casual sex, as can be explained by the theory of parental investment and sexual selection. The literature in the area of evolutionary psychology suggests that, compared to males, viviparity and the development of the placenta placed an important burden of time and energy in females [106]. This differential investment would be responsible for hypothalamic distinctions in the course of evolution, with differential hormonal effects during the development of the brain. It is therefore likely that post-TBI neuroendocrine dysfunction could change the expression of these evolutionary characteristics. However, this interpretation needs to be considered with caution not only because we did not measure hormonal changes, but also because human sexual behavior does not rely only on hormones. Human sexuality is multifactorial and based on psychological traits, behaviors, and cultural specificities, among others. Studies incorporating a more environmen$\mathrm{tal} /$ sociocultural perspective in this area are thus warranted considering the complexity and inherent multidisciplinary nature of sexuality.

Our third main finding is that infidelity levels, with infidelity defined as a person being unfaithful in a committed monogamous relationship, were comparable in healthy controls and individuals with TBI. Also, there were no differences according to sex. Taken together, these results are the first to reveal the nature of attitudes toward infidelity following TBI. It can thus be suggested that attitudes towards infidelity following TBI are not different from those of healthy controls. Therefore, a possible explanation is that, after a TBI, people's attitudes toward infidelity do not change.

In contrast to earlier findings showing that an unrestricted sociosexual orientation is associated with a greater willingness to engage in infidelity [13], the results of the current study could not find evidence of this link. A possible explanation of this might be that we used a general infidelity scale, while Mattingly et al.s study included ambiguous, deceptive, and explicit infidelity [13]. This lack of uniformity in instruments to measure infidelity is one of the challenges regarding research in this area and may be responsible for incongruent findings [2].

Surprisingly, infidelity scores were negatively associated with sociosexual behavior, sociosexual attitudes, and sociosexual desire in individuals with TBI but not in healthy controls. This finding was unexpected and suggests that individuals with TBI reporting low levels in infidelity scores (e.g., disapproving of infidelity) also show high levels of SO (unrestricted SO). This finding indicates that individuals with TBI who express less acceptance of infidelity also report a more promiscuous mating strategy in terms of behavior (e.g., number of sexual partners in the last year), attitudes (e.g., imagining themselves enjoying casual sex with different partners), and desire (e.g., reporting a high frequency of spontaneous sexual fantasies with someone they have just met).

There are several possible explanations for these results. Firstly, individuals with TBI may have problems with emotional regulation that contribute to difficulties to control their own behavior [107]. As a consequence, thinking that being unfaithful in a committed monogamous relationship is not acceptable does not necessarily translate into regulating their own behaviors, attitudes, and desires regarding their willingness to engage in uncommitted sex. In fact, difficulties with behavioral regulation and social cognition are also common symptoms following TBI [108]. Another possible explanation is related to difficulties involving lack of awareness or anosognosia [109]. Anosognosia can affect emotional recognition and the interpretation of social signals [110]. This could explain the existence of this discrepancy where individuals with TBI can have difficulties in integrating what they do with what they think and what they feel. Another possible explanation to address this result can be related to hypersexuality. However, in a multicenter study, the estimated prevalence of inappropriate sexual behaviors following TBI was $8.9 \%$ and particularly evidenced in a minority of younger individuals with more severe injuries [111]. Such an explanation seems to be less probable as the sample of this study included a majority of milder injuries.

Limitations and Future Directions. The current study investigated the relationship between sociosexuality and attitudes towards infidelity following TBI. However, the results should be interpreted with caution in the face of several limitations. First, contrary to the epidemiological data of TBI in Canada, the sample included predominantly women with TBI whilst, regardless of age group, the overall rate of TBI is higher in men than women [112]. However, most of research conducted in sexuality and TBI has an underrepresentation of women [113]; so this could also be interpreted as one of the strengths of our study which included more than $45 \%$ of males. Furthermore, in the current study, $67 \%$ of TBI individuals had a mild TBI. Hence, caution is warranted in generalizing our results to moderate to severe TBI. In consequence, research on sociosexuality and infidelity needs to be conducted in larger samples, in particular with moderate to severe TBI.

Secondly, participants completed self-report measures to describe their sexual behavior. As sex is typically a highly private activity, people can conceal their true sexual behavior in an interview because sometimes they feel intensely embarrassed and threatened and may experience fear of reprisals when asked to reveal their sexual life [114]. However, to increase the validity of self-reported sexual behavior and avoid self-presentation bias, the questionnaires were completed anonymously. The study was conducted in a province that is highly open with respect to sexuality. For example, the results of a study revealed that people living in Quebec were more likely than participants from all other regions of Canada to report an interest in engaging in casual sex [105]. In this respect, our results cannot be extrapolated to other countries with different cultural backgrounds, especially those with more conservative attitudes towards sexuality. Future research should therefore concentrate on the investigation of cultural differences in sociosexuality and attitudes towards infidelity, by carefully controlling for methodological difficulties, such as presentation bias, among others [115].

As a third limitation, the current study was correlational/ cross-sectional; so it was not possible to infer directional relationships between sociosexuality and attitudes towards 
infidelity in this group of individuals with TBI. Consequently, we cannot make inferences about causation and our interpretations should be treated as exploratory hypotheses. Prospective and longitudinal studies with larger samples will allow further more solid study of attitudes towards infidelity. The reasons are twofold: attitudes can change over time and also the relationships between attitudes with other psychological variables can also change with time. More broadly, additional research is required to understand this dynamics. This information would be useful when addressing psychosexual issues in individuals with TBI.

Finally, our study included exclusively a small sample of adults and, as such, results cannot be generalized to teenagers or older adults with TBI. In fact, most research has focused on adult TBI brain-behavior correlates with minimal involvement of adolescents [116]. In consequence, research needs to examine adolescents with TBI regarding sociosexuality and attitudes toward infidelity. Also, it would be interesting to compare experiences of sexually diverse people regarding infidelity and sociosexuality, such as lesbian, gay, bisexual, transgender, and intersex individuals with TBI [117].

As a closing remark, little attention has been given to within-sex individual differences in the type of infidelity found to be more distressing. This was not part of our study objectives. However, it is recommended to explore the hypothesis that greater sexual permissiveness (e.g., higher scores on sociosexuality) is associated with greater distress to sexual infidelity [4]. Sexual and emotional types of infidelity need to be addressed following TBI.

Despite all these limitations, the present study makes a unique contribution to the field of sexuality following TBI. Our study provides additional evidence with respect to sociosexuality after TBI and suggests a possible link between evolutionary psychology and neuropsychological effects of TBI. A better understanding of the interplay between biological, psychological, and sociocultural processes is needed to do justice to the complexity of this subject matter [118], in particular as it is expressed or modified following TBI.

\section{Conclusions}

This paper reports the fact that men with TBI show a trend towards the reduction of sociosexuality levels, suggesting the possible modification of a complex and deeply rooted psychosexual trait after a TBI. In addition, our results confirm that there are statistically significant differences according to sex in sociosexuality, supporting previous evidence that, compared to women, men have higher levels of sociosexuality across cultures. Finally, our findings indicate that although infidelity was comparable in healthy controls and individuals with TBI, individuals with TBI who express less acceptance of infidelity also report a more promiscuous mating strategy in terms of behavior, attitudes, and desire. This work contributes to existing knowledge in the field of sexuality and psychosexual changes following TBI. Taken together, the main theoretical implications correspond to the development of a link between evolutionary psychology and neuropsychology.

\section{Conflict of Interests}

The authors declare that there is no conflict of interests regarding the publication of this paper.

\section{Acknowledgments}

The authors wish to thank the CRIR, CERNEC, and Faculté des études supérieures et postdoctorales de l'Université de Montréal (scholarships to Jhon Alexander Moreno), the CRLB for partial funding of this study (grant to Michelle McKerral), and the participants who took part in the study.

\section{References}

[1] L. Josephs, "The adaptive functions of sexual plasticity: the suppression and surreptitious expression of human sociosexuality," Psychodynamic Psychiatry, vol. 40, no. 2, pp. 243-274, 2012.

[2] A. J. Blow and K. Hartnett, "Infidelity in committed relationships I: a methodological review," Journal of Marital and Family Therapy, vol. 31, no. 2, pp. 183-216, 2005.

[3] A. J. Blow and K. Hartnett, "Infidelity in committed relationships II: a substantive review," Journal of Marital and Family Therapy, vol. 31, no. 2, pp. 217-233, 2005.

[4] S. Treger and S. Sprecher, "The influences of sociosexuality and attachment style on reactions to emotional versus sexual infidelity," Journal of Sex Research, vol. 48, no. 5, pp. 413-422, 2011.

[5] H. Takahashi, M. Matsuura, N. Yahata, M. Koeda, T. Suhara, and Y. Okubo, "Men and women show distinct brain activations during imagery of sexual and emotional infidelity," NeuroImage, vol. 32, no. 3, pp. 1299-1307, 2006.

[6] A. C. Kinsey, Sexual Behavior in the Human Female, Pocket Books, New York, NY, USA, 1965.

[7] A. C. Kinsey, W. Pomeroy, and C. Martin, Sexual Behavior in the Human Male, W.B. Saunders, Philadelphia, Pa, USA, 1948.

[8] J. A. Simpson and S. W. Gangestad, "Individual differences in sociosexuality: evidence for convergent and discriminant validity," Journal of Personality and Social Psychology, vol. 60, no. 6, pp. 870-883, 1991.

[9] D. P. Schmitt, "Sociosexuality from Argentina to Zimbabwe: a 48-nation study of sex, culture, and strategies of human mating," Behavioral and Brain Sciences, vol. 28, no. 2, pp. 247-311, 2005.

[10] R. S. Edelstein, W. J. Chopik, and E. L. Kean, "Sociosexuality moderates the association between testosterone and relationship status in men and women," Hormones and Behavior, vol. 60, no. 3, pp. 248-255, 2011.

[11] M. C. Quist, C. D. Watkins, F. G. Smith, A. C. Little, L. M. Debruine, and B. C. Jones, "Sociosexuality predicts women's preferences for symmetry in men's faces," Archives of Sexual Behavior, vol. 41, no. 6, pp. 1415-1421, 2012.

[12] S. Sprecher, S. Treger, and J. K. Sakaluk, "Premarital sexual standards and sociosexuality: gender, ethnicity, and cohort differences," Archives of Sexual Behavior, vol. 42, no. 8, pp. 1395-1405, 2013.

[13] B. A. Mattingly, E. M. Clark, D. J. Weidler, M. Bullock, J. Hackathorn, and K. Blankmeyer, "Sociosexual orientation, commitment, and infidelity: a mediation analysis," Journal of Social Psychology, vol. 151, no. 3, pp. 222-226, 2011.

[14] T. H. Rammsayer and S. J. Troche, "The relationship between sociosexuality and aspects of body image in men and women: 
a structural equation modeling approach," Archives of Sexual Behavior, vol. 42, no. 7, pp. 1173-1179, 2013.

[15] M. R. Yost and E. L. Zurbriggen, "Gender differences in the enactment of sociosexuality: an examination of implicit social motives, sexual fantasies, coercive sexual attitudes, and aggressive sexual behavior," Journal of Sex Research, vol. 43, no. 2, pp. 163-173, 2006.

[16] J. Hofer, H. Busch, M. H. Bond, D. Campos, M. Li, and R. Law, "The implicit power motive and sociosexuality in men and women: pancultural effects of responsibility," Journal of Personality and Social Psychology, vol. 99, no. 2, pp. 380-394, 2010.

[17] R. Trivers, Natural Selection and Social Theory: Selected Papers of Robert L. Trivers, Evolution and Cognition, Oxford University Press, New York, NY, USA, 2002.

[18] D. M. Buss and D. P. Schmitt, "Sexual strategies theory: an evolutionary perspective on human mating," Psychological Review, vol. 100, no. 2, pp. 204-232, 1993.

[19] R. L. Trivers and D. E. Willard, "Natural selection of parental ability to vary the sex ratio of offspring," Science, vol. 179, no. 4068, pp. 90-92, 1973.

[20] M. Schaller and D. R. Murray, "Pathogens, personality, and culture: disease prevalence predicts worldwide variability in sociosexuality, extraversion, and openness to experience," Journal of Personality and Social Psychology, vol. 95, no. 1, pp. 212-221, 2008.

[21] W. H. A. Ryu, A. Feinstein, A. Colantonio, D. L. Streiner, and D. R. Dawson, "Early identification and incidence of mild TBI in Ontario," Canadian Journal of Neurological Sciences, vol. 36, no. 4, pp. 429-435, 2009.

[22] A. M. Sander and K. L. Maestas, "Sexuality after traumatic brain injury," Archives of Physical Medicine and Rehabilitation, vol. 95, no. 9, pp. 1801-1802, 2014.

[23] A. Moreno, C. Gan, N. Zasler et al., "Neurosexuality: neurobehavioral approaches to the study of sexuality after traumatic brain injury," in Proceedings of the 91st American Congress of Rehabilitation Medicine: Translation to Clinical Practice, Toronto, Canada, 2014.

[24] J. L. Ponsford, M. G. Downing, and R. Stolwyk, "Factors associated with sexuality following traumatic brain injury," The Journal of Head Trauma Rehabilitation, vol. 28, no. 3, pp. 195201, 2013.

[25] J. A. Moreno, J. C. Arango Lasprilla, C. Gan, and M. Mckerral, "Sexuality after traumatic brain injury: a critical review," NeuroRehabilitation, vol. 32, no. 1, pp. 69-85, 2013.

[26] M. G. Downing, R. Stolwyk, and J. L. Ponsford, "Sexual changes in individuals with traumatic brain injury: a control comparison," Journal of Head Trauma Rehabilitation, vol. 28, no. 3, pp. 171-178, 2013.

[27] A. Moreno, M. McKerral, and C. Gan, "Neurosexuality: a biopsychosocial model to conceptualize psychosexual difficulties after traumatic brain injury," in Proceedings of the $34 \mathrm{rd}$ Interamerican Congress of Psychology: Knowledge, Diversity and Integration, Brasilia, Brazil, 2013.

[28] G. Simpson, "Addressing the sexual concerns of persons with traumatic brain injury in rehabilitation settings: a framework for action," Brain Impairment, vol. 2, no. 2, pp. 97-108, 2001.

[29] M. Kreuter, A.-G. Dahllöf, G. Gudjonsson, M. Sullivan, and A. Siösteen, "Sexual adjustment and its predictors after traumatic brain injury," Brain Injury, vol. 12, no. 5, pp. 349-368, 1998.
[30] R. E. O'carroll, J. Woodrow, and F. Maroun, "Psychosexual and psychosocial sequelae of closed head injury," Brain Injury, vol. 5, no. 3, pp. 303-313, 1991.

[31] M. E. Sandel, R. Delmonico, and M. J. Kotch, "Sexuality, reproduction, and neuroendocrine disorders following TBI," in Brain Injury Medicine: Principles and Practice, N. D. Zasler, D. I. Katz, and R. D. Zafonte, Eds., pp. 673-695, Demos, New York, NY, USA, 2007.

[32] M. Cohen, "Physical and medical aspects of sexuality after traumatic brain injury," in Sexual Difficulties After Traumatic Brain Injury and Ways to Deal With It, R. Aloni and S. Katz, Eds., pp. 42-56, Charles C Thomas, Springfield, Ill, USA, 2003.

[33] A. M. Sander, K. L. Maestas, T. G. Nick et al., "Predictors of sexual functioning and satisfaction 1 year following traumatic brain injury: a TBI model systems multicenter study," The Journal of Head Trauma Rehabilitation, vol. 28, no. 3, pp. 186194, 2013.

[34] M. E. Sandel, R. Delmonico, and M. J. Kotch, "Sexuality and intimacy following traumatic brain injury," in Brain Injury Medicine: Principles and Practice, N. Zasler and R. Zafonte, Eds., pp. 954-973, Demos Publishers, New York, NY, USA, 2013.

[35] Gouvernement du Québec and Ministère de la Santé et des Services Sociaux, Orientations Ministérielles pour le TCCL, Gouvernement du Québec, Ministère de la Santé et des Services Sociaux, 2005.

[36] C. M. Davis, Handbook of Sexuality-Related Measures, Sage Publications, Thousand Oaks, Calif, USA, 1998.

[37] L. Penke and J. B. Asendorpf, "Beyond global sociosexual orientations: a more differentiated look at sociosexuality and its effects on courtship and romantic relationships," Journal of Personality and Social Psychology, vol. 95, no. 5, pp. 1113-1135, 2008.

[38] K. Wilson, B. A. Mattingly, E. M. Clark, D. J. Weidler, and A. W. Bequette, "The gray area: exploring attitudes toward infidelity and the development of the perceptions of dating infidelity scale," The Journal of Social Psychology, vol. 151, no. 1, pp. 63-86, 2011.

[39] D. T. A. Eisenberg, B. Campbell, J. MacKillop, J. K. Lum, and D. S. Wilson, "Season of birth and dopamine receptor gene associations with impulsivity, sensation seeking and reproductive behaviors," PLoS ONE, vol. 2, no. 11, Article ID e1216, 2007.

[40] L. A. Duncan, J. H. Park, J. Faulkner, M. Schaller, S. L. Neuberg, and D. T. Kenrick, "Adaptive allocation of attention: effects of sex and sociosexuality on visual attention to attractive oppositesex faces," Evolution and Human Behavior, vol. 28, no. 5, pp. 359-364, 2007.

[41] J. S. Allen and K. G. Bailey, "Are mating strategies and mating tactics independent constructs?" Journal of Sex Research, vol. 44, no. 3, pp. 225-232, 2007.

[42] M. McIntyre, S. W. Gangestad, P. B. Gray et al., "Romantic involvement often reduces men's testosterone levels-but not always: the moderating role of extrapair sexual interest," Journal of Personality and Social Psychology, vol. 91, no. 4, pp. 642-651, 2006.

[43] J. M. Townsend and T. Wasserman, "The perception of sexual attractiveness: sex differences in variability," Archives of Sexual Behavior, vol. 26, no. 3, pp. 243-268, 1997.

[44] D. W. Seal and G. Agostinelli, "Individual differences associated with high-risk sexual behaviour: implications for intervention programmes," AIDS Care: Psychological and Socio-medical Aspects of AIDS/HIV, vol. 6, no. 4, pp. 393-397, 1994. 
[45] D. Knox, M. E. Zusman, M. Kaluzny, and L. Sturdivant, "Attitudes and behavior of college students toward infidelity," College Student Journal, vol. 34, no. 2, pp. 162-165, 2000.

[46] D. Barthel, C. Barkmann, S. Ehrhardt, and C. Bindt, "Psychometric properties of the 7-item Generalized Anxiety Disorder scale in antepartum women from Ghana and Côte d'Ivoire," Journal of Affective Disorders, vol. 169, pp. 203-211, 2014.

[47] IBM, SPSS Advanced Statistics 21, IBM Corporation, 2012.

[48] J. Cohen, Statistical Power Analysis for the Behavioral Sciences, Academic Press, New York, NY, USA, 1969.

[49] C. Waterhouse, "Post-traumatic hypopituitarism (PTHP): an under-diagnosed complication," British Journal of Neuroscience Nursing, vol. 8, no. 4, pp. 184-190, 2012.

[50] F. Tanriverdi, A. De Bellis, H. Ulutabanca et al., "A five year prospective investigation of anterior pituitary function after traumatic brain injury: is hypopituitarism long-term after head trauma associated with autoimmunity?" Journal of Neurotrauma, vol. 30, no. 16, pp. 1426-1433, 2013.

[51] F. Prodam, V. Gasco, M. Caputo et al., "Metabolic alterations in patients who develop traumatic brain injury (TBI)-induced hypopituitarism," Growth Hormone and IGF Research, vol. 23, no. 4, pp. 109-113, 2014.

[52] N. L. Heather, C. Jefferies, P. L. Hofman et al., "Permanent hypopituitarism is rare after structural traumatic brain injury in early childhood," The Journal of Clinical Endocrinology and Metabolism, vol. 97, no. 2, pp. 599-604, 2012.

[53] F. Tanriverdi, A. Agha, G. Aimaretti et al., "Manifesto for the current understanding and management of traumatic brain injury-induced hypopituitarism," Journal of Endocrinological Investigation, vol. 34, no. 7, pp. 541-543, 2011.

[54] H. J. Schneider, M. Schneider, I. Kreitschmann-Andermahr et al., "Structured assessment of hypopituitarism after traumatic brain injury and aneurysmal subarachnoid hemorrhage in 1242 patients: the German interdisciplinary database," Journal of Neurotrauma, vol. 28, no. 9, pp. 1693-1698, 2011.

[55] I. Kreitschmann-Andermahr, Y. Hartmann, E. Poll, H. J. Schneider, M. Buchfelder, and G. K. Stalla, "The German database on hypopituitarism after traumatic brain injury and aneurysmal subarachnoid hemorrhage-description, objectives and design," Experimental and Clinical Endocrinology \& Diabetes, vol. 119, no. 1, pp. 15-20, 2011.

[56] N. E. Kokshoorn, J. W. A. Smit, W. A. Nieuwlaat et al., "Low prevalence of hypopituitarism after traumatic brain injury: a multicenter study," European Journal of Endocrinology, vol. 165, no. 2, pp. 225-231, 2011.

[57] E. Fernandez-Rodriguez, I. Bernabeu, A. I. Castro, F. Kelestimur, and F. F. Casanueva, "Hypopituitarism following traumatic brain injury: determining factors for diagnosis," Frontiers in Endocrinology, vol. 2, article 25, 2010.

[58] F. Tanriverdi, K. Unluhizarci, and F. Kelestrimur, "Persistent neuroinflammation may be involved in the pathogenesis of traumatic brain injury (TBI)-induced hypopituitarism: Potential genetic and autoimmune factors," Journal of Neurotrauma, vol. 27, no. 2, pp. 301-302, 2010.

[59] S. Kolli, A. Mallipedhi, T. Hughes, and P. Evans, "Delayed diagnosis of hypopituitarism following severe traumatic brain injury," BMJ Case Reports, 2010.

[60] N. E. Kokshoorn, M. J. E. Wassenaar, N. R. Biermasz et al., "Hypopituitarism following traumatic brain injury: prevalence is affected by the use of different dynamic tests and different normal values," European Journal of Endocrinology, vol. 162, no. 1, pp. 11-18, 2010.
[61] A. F. Guerrero and A. Alfonso, “Traumatic brain injury-related hypopituitarism: a review and recommendations for screening combat veterans," Military Medicine, vol. 175, no. 8, pp. 574-580, 2010.

[62] H. Valdes-Socin, L. Vroonen, P. Robe et al., "[Traumatic brain injury and subarachnoid hemorrhage as a cause of hypopituitarism: a review]. Hypopituitarisme consécutif aux atteintes cérébrales: le traumatisme crânien et l'hémorragie sousarachnoïdienne mis en cause," Revue Médicale de Liège, vol. 64, no. 9, pp. 457-463, 2009.

[63] L. Srinivasan, B. Roberts, T. Bushnik et al., "The impact of hypopituitarism on function and performance in subjects with recent history of traumatic brain injury and aneurysmal subarachnoid haemorrhage," Brain Injury, vol. 23, no. 7-8, pp. 639648, 2009.

[64] T. Saito, N. Sato, M. Kimoto et al., "Incomplete deficiency of hypothalamic hormones in hypothalamic hypopituitarism associated with an old traumatic brain injury," Endocrine Journal, vol. 56, no. 8, pp. 945-950, 2009.

[65] D. D. Makulski, K. H. Taber, and F. Y. Chiou-Tan, "Neuroimaging in posttraumatic hypopituitarism," Journal of Computer Assisted Tomography, vol. 32, no. 2, pp. 324-328, 2008.

[66] A. Liew and C. J. Thompson, "Is hypopituitarism predictable after traumatic brain injury?" Nature Clinical Practice Endocrinology \& Metabolism, vol. 4, no. 3, pp. 126-127, 2008.

[67] M. Klose and U. Feldt-Rasmussen, "Does the type and severity of brain injury predict hypothalamo-pituitary dysfunction? Does post-traumatic hypopituitarism predict worse outcome?" Pituitary, vol. 11, no. 3, pp. 255-261, 2008.

[68] S. Bavisetty, S. Bavisetty, D. L. McArthur, and et al, "Chronic hypopituitarism after traumatic brain injury: risk assessment and relationship to outcome," Neurosurgery, vol. 62, no. 5, pp. 1080-1094, 2008.

[69] F. Tanriverdi, K. Unluhizarci, B. Coksevim, A. Selcuklu, F. F. Casanueva, and F. Kelestimur, "Kickboxing sport as a new cause of traumatic brain injury-mediated hypopituitarism," Clinical Endocrinology, vol. 66, no. 3, pp. 360-366, 2007.

[70] H. J. Schneider, P. G. Sämann, M. Schneider et al., "Pituitary imaging abnormalities in patients with and without hypopituitarism after traumatic brain injury," Journal of Endocrinological Investigation, vol. 30, no. 4, pp. RC9-RC12, 2007.

[71] H. J. Schneider, G. Corneli, I. Kreitschman-Andermahr et al., "Traumatic brain injury and hypopituitarism in children and adolescents: is the problem under-estimated?" Pediatric Endocrinology Reviews, vol. 4, no. 3, pp. 205-209, 2007.

[72] M. Medic-Stojanoska, S. Pekic, N. Curic, D. Djilas-Ivanovic, and V. Popovic, "Evolving hypopituitarism as a consequence of traumatic brain injury (TBI) in childhood-call for attention," Endocrine, vol. 31, no. 3, pp. 268-271, 2007.

[73] E. Ghigo, "Hypopituitarism following traumatic brain injury," Hormone Research, vol. 68, no. 5, pp. 12-13, 2007.

[74] G. Corneli, E. Ghigo, and G. Aimaretti, "Managing patients with hypopituitarism after traumatic brain injury," Current Opinion in Endocrinology, Diabetes and Obesity, vol. 14, no. 4, pp. 301305, 2007.

[75] A. Agha, J. Phillips, and C. J. Thompson, "Hypopituitarism following traumatic brain injury (TBI)," British Journal of Neurosurgery, vol. 21, no. 2, pp. 210-216, 2007.

[76] F. Tanriverdi, H. Senyurek, K. Unluhizarci, A. Selcuklu, F. F. Casanueva, and F. Kelestimur, "High risk of hypopituitarism after traumatic brain injury: a prospective investigation of anterior pituitary function in the acute phase and 12 months after 
trauma," The Journal of Clinical Endocrinology and Metabolism, vol. 91, no. 6, pp. 2105-2111, 2006.

[77] H. J. Schneider, G. K. Stalla, and M. Buchfelder, "Expert meeting: hypopituitarism after traumatic brain injury and subarachnoid haemorrhage," Acta Neurochirurgica, vol. 148, no. 4, pp. 449-456, 2006.

[78] B. L. Herrmann, J. Rehder, S. Kahlke et al., "Hypopituitarism following severe traumatic brain injury," Experimental and Clinical Endocrinology and Diabetes, vol. 114, no. 6, pp. 316-321, 2006.

[79] Y.-C. Chang, J.-C. Tsai, and F.-Y. Tseng, "Neuropsychiatric disturbances and hypopituitarism after traumatic brain injury in an elderly man," Journal of the Formosan Medical Association, vol. 105, no. 2, pp. 172-176, 2006.

[80] C. L. Acerini, R. C. Tasker, S. Bellone, G. Bona, C. J. Thompson, and M. O. Savage, "Hypopituitarism in childhood and adolescence following traumatic brain injury: the case for prospective endocrine investigation," European Journal of Endocrinology, vol. 155, no. 5, pp. 663-669, 2006.

[81] R. J. Urban, P. Harris, and B. Masel, "Anterior hypopituitarism following traumatic brain injury," Brain Injury, vol. 19, no. 5, pp. 349-358, 2005.

[82] V. Popovic, G. Aimaretti, F. F. Casanueva, and E. Ghigo, "Hypopituitarism following traumatic brain injury," Frontiers of Hormone Research, vol. 33, pp. 33-44, 2005.

[83] V. Popovic, G. Aimaretti, F. F. Casanueva, and E. Ghigo, "Hypopituitarism following traumatic brain injury (TBI): call for attention," Journal of Endocrinological Investigation, vol. 28, no. 5, supplement, pp. 61-64, 2005.

[84] B. E. Masel, "Traumatic brain injury induced hypopituitarism: the need and hope of rehabilitation," Pituitary, vol. 8, no. 3-4, pp. 263-266, 2005.

[85] M. Lorenzo, R. Peino, A. I. Castro et al., "Hypopituitarism and growth hormone deficiency in adult subjects after traumatic brain injury: who and when to test," Pituitary, vol. 8, no. 3-4, pp. 233-237, 2005.

[86] A. Leal-Cerro, J. M. Florest, M. Rincon et al., "Prevalence of hypopituitarism and growth hormone deficiency in adults longterm after severe traumatic brain injury," Clinical Endocrinology, vol. 62, no. 5, pp. 525-532, 2005.

[87] E. Ghigo, B. Masel, G. Aimaretti et al., "Consensus guidelines on screening for hypopituitarism following traumatic brain injury," Brain Injury, vol. 19, no. 9, pp. 711-724, 2005.

[88] M. Bondanelli, M. R. Ambrosio, M. C. Zatelli, L. de Marinis, and E. C. degli Uberti, "Hypopituitarism after traumatic brain injury," European Journal of Endocrinology, vol. 152, no. 5, pp. 679-691, 2005.

[89] P. Beck-Peccoz, "Is hypopituitarism a common event following traumatic brain injury?" Nature Clinical Practice Endocrinology \& Metabolism, vol. 1, no. 1, pp. 12-13, 2005.

[90] R. Baldelli, S. Bellone, G. Corneli, S. Savastio, A. Petri, and G. Bona, "Traumatic brain injury-induced hypopituitarism in adolescence," Pituitary, vol. 8, no. 3-4, pp. 255-257, 2005.

[91] G. Aimaretti and E. Ghigo, "Traumatic brain injury and hypopituitarism," TheScientificWorldJOURNAL, vol. 5, pp. 777-781, 2005.

[92] G. Aimaretti, M. R. Ambrosio, C. Di Somma et al., "Hypopituitarism induced by traumatic brain injury in the transition phase," Journal of Endocrinological Investigation, vol. 28, no. 11, pp. 984-989, 2005.
[93] V. Popovic, S. Pekic, D. Pavlovic et al., "Hypopituitarism as a consequence of traumatic brain injury (TBI) and its possible relation with cognitive disabilities and mental distress," Journal of Endocrinological Investigation, vol. 27, no. 11, pp. 1048-1054, 2004.

[94] B. E. Masel, "Rehabilitation and hypopituitarism after traumatic brain injury," Growth Hormone \& IGF Research, vol. 14, supplement, pp. S108-S113, 2004.

[95] F. F. Casanueva, E. Ghigo,, and V. Popovic, "Hypopituitarism following traumatic brain injury (TBI): a guideline decalogue," Journal of Endocrinological Investigation, vol. 27, no. 8, pp. 793795, 2004.

[96] G. Aimaretti, M. R. Ambrosio, S. Benvenga et al., "Hypopituitarism and growth hormone deficiency (GHD) after traumatic brain injury (TBI)," Growth Hormone \& IGF Research, vol. 14, supplement, pp. S114-S117, 2004.

[97] A. Mitchell, N. Steffenson, and K. Davenport, "Hypopituitarism due to traumatic brain injury: a case study," Critical Care Nurse, vol. 17, no. 4, pp. 34-54, 1997.

[98] O. M. Edwards and J. D. A. Clark, "Post-traumatic hypopituitarism. Six cases and a review of the literature," Medicine, vol. 65, no. 5, pp. 281-290, 1986.

[99] N. D. Zasler and M. F. Martelli, "Sexual dysfunction," in Textbook of Traumatic Brain Injury, J. M. Silver, W. B. McAllister, and S. C. Yudofsky, Eds., pp. 397-414, American Psychiatric Publishing, Washington, DC, USA, 2011.

[100] T. Greco, D. A. Hovda, and M. L. Prins, "Adolescent TBIinduced hypopituitarism causes sexual dysfunction in adult male rats," Developmental Neurobiology, vol. 75, no. 2, pp. 193202, 2015.

[101] W. J. Zheng, X. D. Zhou, X. L. Wang, and T. Hesketh, "Sociosexuality in mainland China," Archives of Sexual Behavior, vol. 43, no. 3, pp. 621-629, 2014.

[102] C. P. Cross, "Sex differences in same-sex direct aggression and sociosexuality: the role of risky impulsivity," Evolutionary Psychology, vol. 8, no. 4, pp. 779-792, 2010.

[103] R. A. Lippa, "Sex differences in sex drive, sociosexuality, and height across 53 nations: testing evolutionary and social structural theories," Archives of Sexual Behavior, vol. 38, no. 5, pp. 631-651, 2009.

[104] J. A. Simpson and S. W. Gangestad, "Sociosexuality and romantic partner choice," Journal of Personality, vol. 60, no. 1, pp. 31-51, 1992.

[105] D. S. Fischtein, E. S. Herold, and S. Desmarais, "How much does gender explain in sexual attitudes and behaviors? A survey of Canadian adults," Archives of Sexual Behavior, vol. 36, no. 3, pp. 451-461, 2007.

[106] E. B. Keverne, "Significance of epigenetics for understanding brain development, brain evolution and behaviour," Neuroscience, vol. 264, pp. 207-217, 2014.

[107] C. Cattran, M. Oddy, and R. Wood, "The development of a measure of emotional regulation following acquired brain injury," Journal of Clinical and Experimental Neuropsychology, vol. 33, no. 6, pp. 672-679, 2011.

[108] T. Manly and F. C. Murphy, "Rehabilitation of executive function and social cognition impairments after brain injury," Current Opinion in Neurology, vol. 25, no. 6, pp. 656-661, 2012.

[109] J. F. Goldberg, L. D. McLeod, S. E. Fehnel, V. S. L. Williams, L. R. Hamm, and K. Gilchrist, "Development and psychometric evaluation of the bipolar functional status questionnaire (bfsq)," Bipolar Disorders, vol. 12, no. 1, pp. 32-44, 2010. 
[110] J. M. Spikman, M. V. Milders, A. C. Visser-Keizer, H. J. Westerhof-Evers, M. Herben-Dekker, and J. van der Naalt, "Deficits in facial emotion recognition indicate behavioral changes and impaired self-awareness after moderate to severe traumatic brain injury," PLoS ONE, vol. 8, no. 6, Article ID e65581, 2013.

[111] G. K. Simpson, M. Sabaz, and M. Daher, "Prevalence, clinical features, and correlates of inappropriate sexual behavior after traumatic brain injury: a multicenter study," Journal of Head Trauma Rehabilitation, vol. 28, no. 3, pp. 202-210, 2013.

[112] Government of Canada and Public Health Agency of Canada, Mapping Connections: An Understanding of Neurological Conditions in Canada, Government of Canada, Public Health Agency of Canada, 2014.

[113] A. Colantonio, J. E. Harris, G. Ratcliff, S. Chase, and K. Ellis, "Gender differences in self reported long term outcomes following moderate to severe traumatic brain injury," $B M C$ Neurology, vol. 10, article 102, 2010.

[114] J. A. Catania, D. R. Gibson, D. D. Chitwood, and T. J. Coates, "Methodological problems in AIDS behavioral research: influences on measurement error and participation bias in studies of sexual behavior," Psychological Bulletin, vol. 108, no. 3, pp. 339362, 1990.

[115] A. A. Stone, The Science of Self-Report: Implications for Research and Practice, Lawrence Erlbaum Associates, Mahwah, NJ, USA, 1999.

[116] R. das Nair, G. Talbot, G. Hughes, and A. Starza-Smith, "Sexuality in adolescents following traumatic brain injury: a systematic review of literature and research proposal," Journal of the International Neuropsychological Society, vol. 13, p. 91, 2007.

[117] A. Moreno and M. McKerral, "Sexual diversity and disability: future directions in traumatic brain injury," International Journal of Psychology, vol. 47, no. 1, p. 684, 2012.

[118] S. Choudhury and J. Slaby, Critical Neuroscience: A Handbook of the Social and Cultural Contexts of Neuroscience, Wiley Blackwell, New York, NY, USA, 2012. 


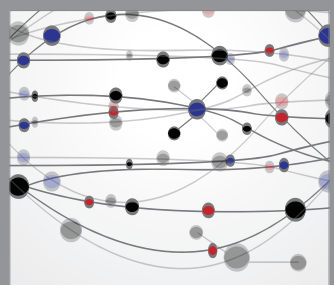

The Scientific World Journal
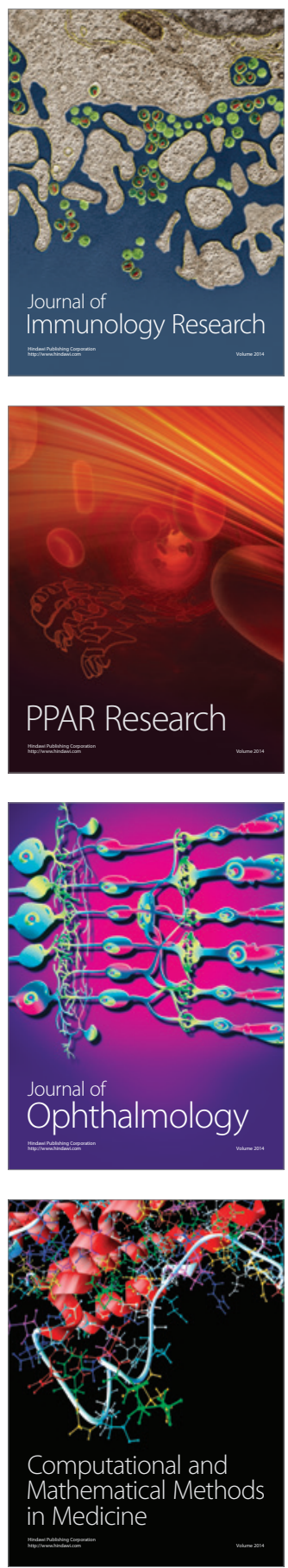

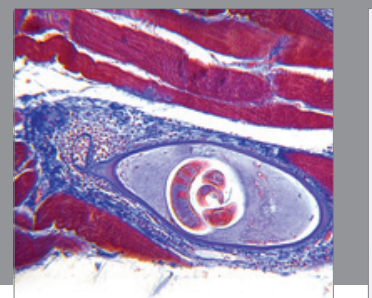

Gastroenterology

Research and Practice
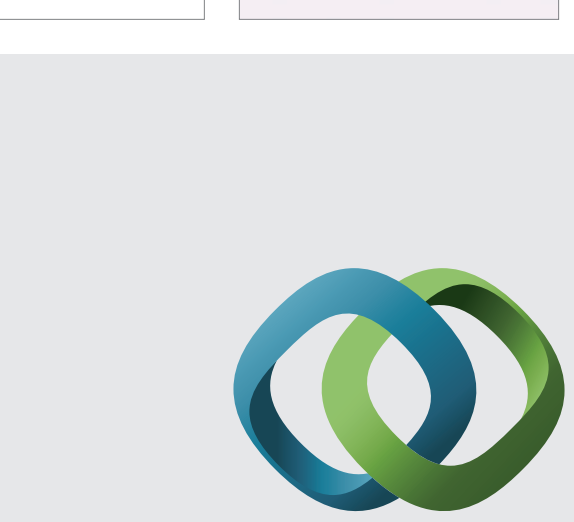

\section{Hindawi}

Submit your manuscripts at

http://www.hindawi.com
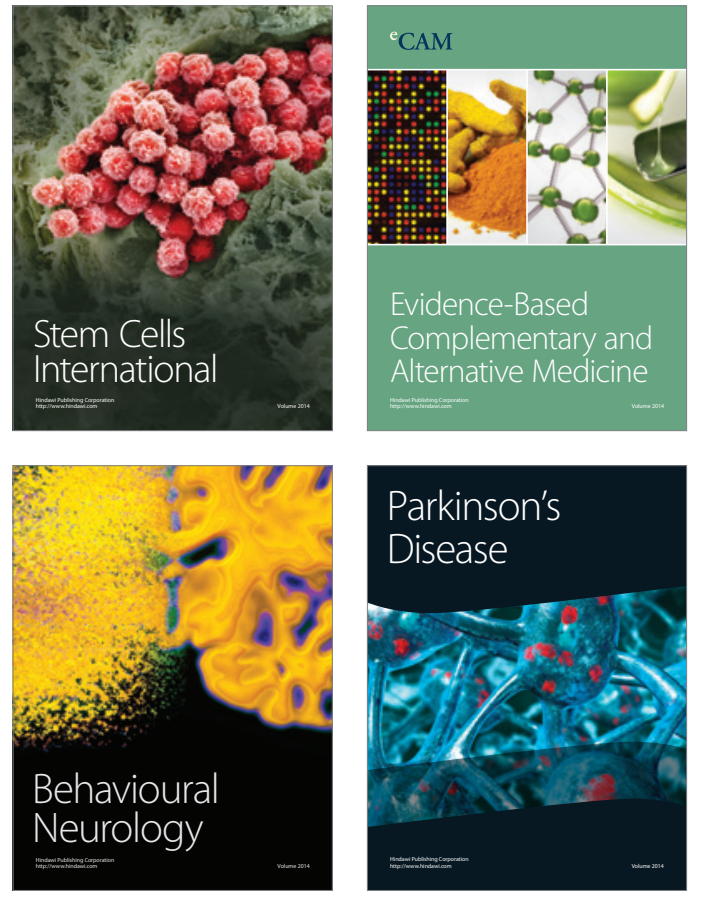
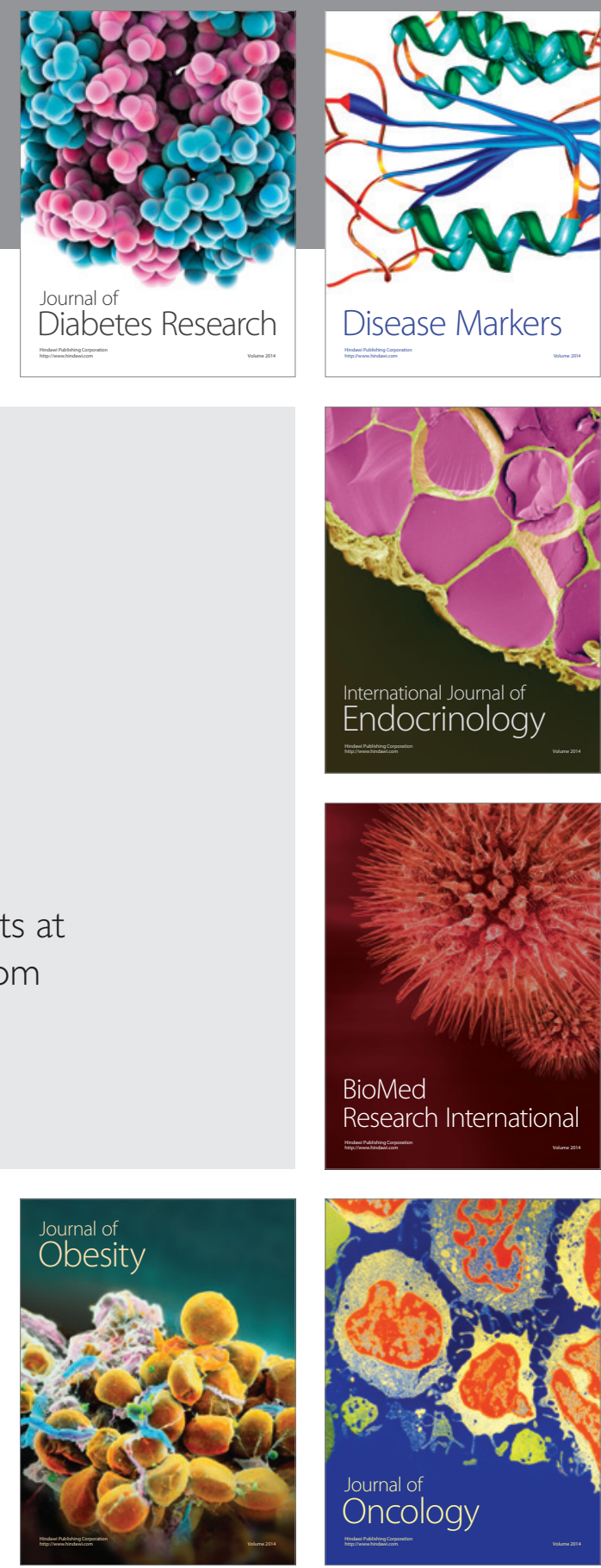

Disease Markers
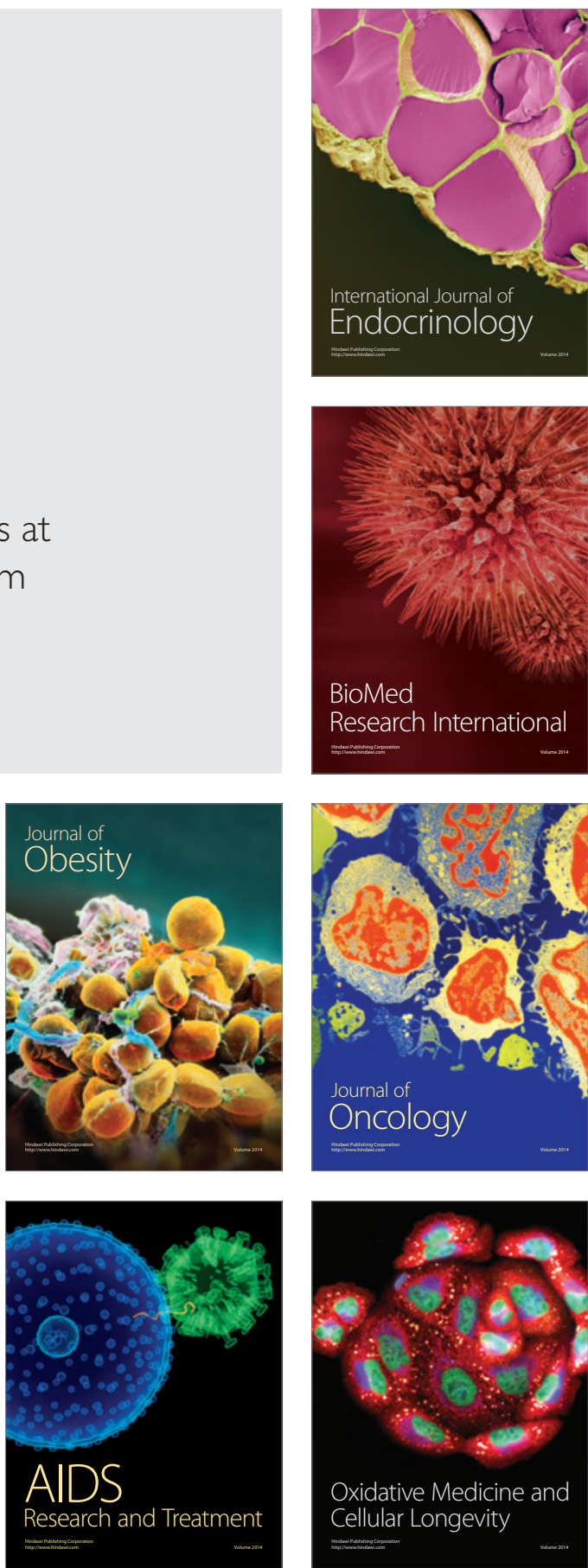